University of Nebraska - Lincoln

DigitalCommons@University of Nebraska - Lincoln

Public Health Resources

Public Health Resources

6-1-2021

\title{
Promise and complexity of lupus mouse models
}

\author{
Erica Moore \\ Albert Einstein College of Medicine of Yeshiva University, erica.moore@einsteinmed.org \\ Joshua A. Reynolds \\ Albert Einstein College of Medicine of Yeshiva University \\ Anne Davidson \\ Feinstein Institutes for Medical Research \\ Stefania Gallucci \\ Lewis Katz School of Medicine \\ Laurence Morel \\ University of Florida College of Medicine
}

See next page for additional authors

Follow this and additional works at: https://digitalcommons.unl.edu/publichealthresources

Part of the Medicine and Health Sciences Commons

Moore, Erica; Reynolds, Joshua A.; Davidson, Anne; Gallucci, Stefania; Morel, Laurence; Rao, Deepak A.; Young, Howard A.; and Putterman, Chaim, "Promise and complexity of lupus mouse models" (2021). Public Health Resources. 565.

https://digitalcommons.unl.edu/publichealthresources/565

This Article is brought to you for free and open access by the Public Health Resources at DigitalCommons@University of Nebraska - Lincoln. It has been accepted for inclusion in Public Health Resources by an authorized administrator of DigitalCommons@University of Nebraska - Lincoln. 


\section{Authors}

Erica Moore, Joshua A. Reynolds, Anne Davidson, Stefania Gallucci, Laurence Morel, Deepak A. Rao, Howard A. Young, and Chaim Putterman 


\title{
Promise and complexity of lupus mouse models
}

\author{
As a follow up to a 2010 meeting deliberating on the benefits of studying mouse models of systemic lupus \\ erythematosus (SLE), the virtual conference "Mouse models of lupus 10 years later" convened on 10 December \\ 2020 to address a challenging decade that saw few new therapies approved, despite leaps in knowledge.
}

A bnormalities in both innate and adaptive immunity characterize SLE, a systemic autoimmune disease with potentially severe consequences in patients. Treatment has traditionally been limited to broad-acting, side-effect-heavy immunosuppressants, which are also incompletely effective. Thus, the need for specific therapies targeting the pathogenic mechanisms of SLE remains unmet. Affirming the utility of mouse models in lupus research, a meeting convened in 2010 recommended that the next decade's research embrace these for their mechanistic insights; their diversity of phenotypes, which mirrors the heterogeneity observed among patients with lupus; and as a platform for clinical exploration. A decade later, hope persists; studies of murine models of lupus have uncovered putative therapies, and many were moved to clinical trials. Unfortunately, few interventions improved patient outcomes: belimumab was the sole therapy brought to market in the 2010s. The continuing difficulties in translating potential into success ${ }^{1-3}$ prompted a second conference, which was recorded.

On 10 December 2020, leading scientists and clinicians from all over the world, in partnership with the National Institutes of Health and the National Cancer Institute, gathered virtually. Hans-Joachim Anders (Ludwig Maximilian University of Munich) sparked much discussion with his opening address highlighting the multiple clinical trial design, diagnostic and scientific pitfalls that have seriously hindered translating success in lupus models to humans. Four scientific sessions followed, each delving into recent basic and translational advances in our understanding and treatment of SLE. In his closing keynote talk, Eric Morand (Monash University) proposed that the recent study of the interferon signature in SLE and the interventions it produced be used as a model for moving between human studies and mouse models, while stressing the scientific considerations needed to successfully utilize lupus models. Through discussions during the 2020 conference, participants aimed to revisit the utility of mouse models in the study of lupus by answering the following questions: Have animal models expanded our understanding

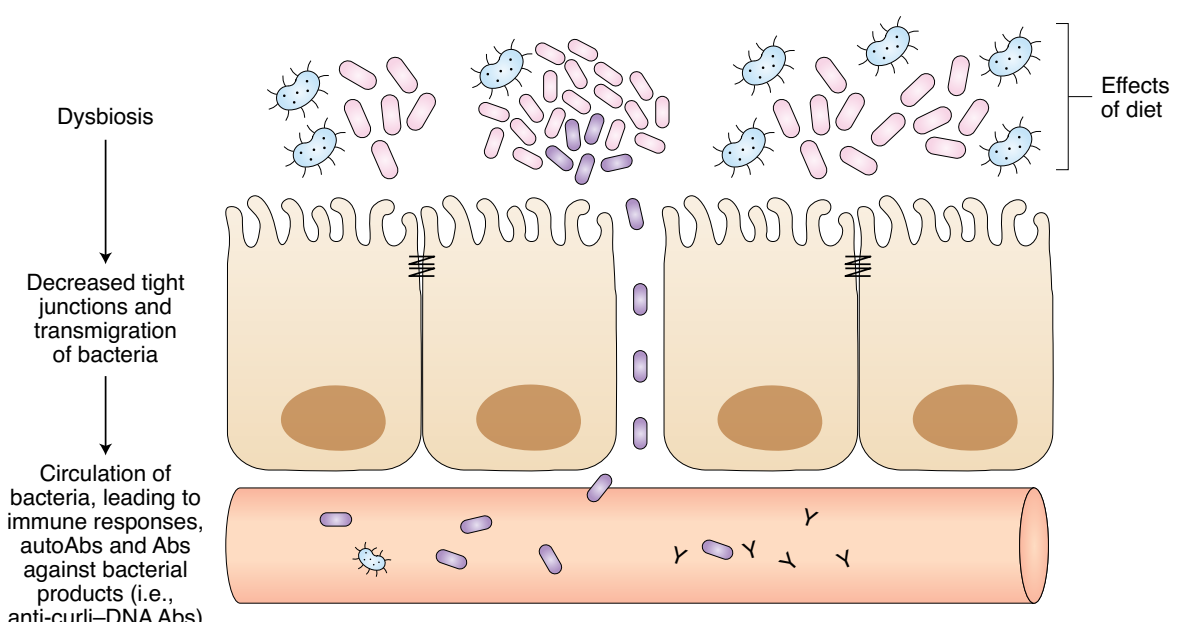

Fig. 1 | Dysbiosis and gut permeability contribute to lupus disease. Expansion of pathogenic gut microbiota can lead to increased permeability of the gut epithelial barrier, providing a route through which bacteria and bacterial antigens can enter systemic circulation. The immune response to these foreign epitopes may generate self-reactive antibodies, such as anti-curli-DNA, and contribute to lupus pathology.

of lupus pathogenesis? In what ways can we target the mediators of the lupus immune response? How has advanced technology been merged with the study of both lupus models and patients with lupus?

\section{Infection and microbiome}

Extraneous infection and the resident microbiome both act as routes through which a predisposed immune system may be hyperstimulated to precipitate loss of tolerance and trigger autoimmunity (Fig. 1). Mark Shlomchik (University of Pittsburgh) reported that experimental autoimmunity following Salmonella infection results from marked extrafollicular expansion of B cell populations, and the rapid maturation of $B$ cells outside germinal centers increases autoantibody levels ${ }^{4}$. Interleukin (IL)-12 mediates this extrafollicular-dominant autoimmune "storm" by suppressing follicular helper $\mathrm{T}\left(\mathrm{T}_{\mathrm{FH}}\right)$ cells necessary for germinal center formation ${ }^{5}$, suggesting that IL-12 inhibition could treat patients with lupus who have similar immune responses.

Stefania Gallucci and Çağla Tükel (Temple University) introduced a specific microbial driver of autoantibody production. The amyloid-nucleic acid compound curli-DNA, an Enterobacteriaceae biofilm component, is found throughout the murine intestinal tract during Salmonella infection. Tükel provided evidence that curli-DNA may trigger autoimmunity once it enters the blood via the intestinal epithelia or when it is shed by biofilm-bearing catheters ${ }^{6}$. Gallucci's group supported this hypothesis, showing that intraperitoneal injection of curli-DNA in lupus NZB $\times W / F 1$ mice increased circulating autoantibody and type I interferon (IFN) concentrations and activated macrophages and dendritic cells ${ }^{7}$. In addition to these hallmarks of SLE, curli-DNA appeared to induce B cell class switching independently of T cells. Finally, Gallucci described circulating anti-curli-DNA antibodies whose levels correlate with flares of active disease in patients with lupus ${ }^{8}$.

Supporting the hypothesis of a defective intestinal barrier exposing the immune system to microbiota and their products, Gregg Silverman (New York University) presented analyses of stool samples from patients with SLE that showed evidence of a more permeable gut. Additionally, his group characterized a new dysbiosis in patients with SLE, with skewing toward more Ruminococcus gnavus bacteria and 


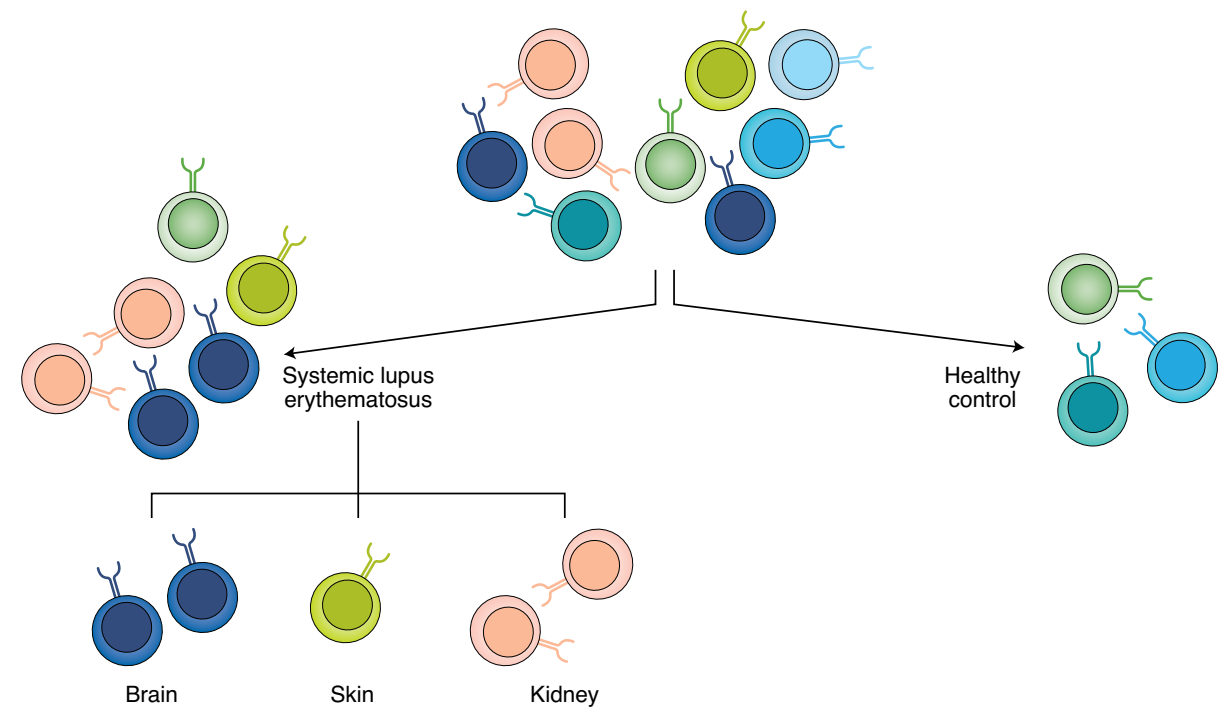

Fig. 2 | The potential application of T cell phenotyping and TCR sequence monitoring at both the organ and disease levels. T cells play a key role in organ damage caused by lupus disease. Sequencing of a $T$ cell receptor could potentially reveal its cognate antigen, including autoantigens. TCRs can be disease and organ specific, so monitoring a patient's TCR repertoire could not only enable a lupus diagnosis but could also reveal organs potentially involved in the patient's disease.

fewer protective species. Serology from these patients showed antibodies that react to a bacterial cell wall lipoglycan, which correlated with active renal disease ${ }^{9}$. With this knowledge, Silverman's group colonized germ-free C57BL/6 mice with $R$. gnavus bacteria and saw levels of serum $R$. gnavus DNA rise, accompanied by elevations in gut permeability and zonulin. Administration of larazotide, a tight-junction regulator, reduced gut permeability, suggesting that $R$. gnavus induces elevated zonulin and disrupts tight junctions to increase gut permeability.

Similarly, Martin Kriegel (Yale University) presented evidence from both mice and human studies that Enterococcus gallinarum induces a more permeable gut, enabling its transmigration to the liver and lymph nodes. Significant immune activation, including rising type I IFN levels and anti-E. gallinarum antibodies, accompanies this process ${ }^{10}$. Interestingly, Kriegel's group developed a vaccine that is able to suppress transmigration of E. gallinarum, which could be a new treatment strategy for autoimmunity related to microbiome dysbiosis.

Xin Luo (Virginia Tech University) characterized the microbiome of diseased $\mathrm{MRL} /$ pr mice; more Lachnospiraceae and fewer Lactobacillaceae were found ${ }^{11}$, representing another pathogenic dysbiosis. The putatively protective Lactobacillaceae were then gavage delivered to female MRL/lpr mice, resulting in improved systemic and renal disease later in life ${ }^{12}$. Oral administration of vancomycin lessened or worsened lupus whether given before or after disease onset, respectively, suggesting different mechanistic effects on the immune system ${ }^{13}$.

Daniel Zegarra-Ruiz (Memorial Sloan Kettering Cancer Center) presented additional findings that point to diet as a contributing factor in murine dysbiosis and lupus. High fiber content in the diets of Toll-like receptor 7-transgenic mice resolved the dysbiosis that contributed to increased gut permeability and a lupus phenotype ${ }^{14}$.

The roles of infection, the microbiome, gut permeability and systemic bacterial products in lupus pathogenesis are only just being appreciated; nevertheless, strong evidence points to robust and far-reaching effects of dysbiosis in mice and humans who are predisposed. While it is impractical to sterilize and then specifically reconstitute the human microbiome, researchers may longitudinally characterize and manipulate a simulated human microbiome in mice as well as evaluate its relationship with disease onset. Mouse models, therefore, are fundamental to advancing our understanding of the interaction between bacteria and the immune system in SLE.

\section{Identifying and repurposing drug targets} Recent advances presented at the meeting identified new drug targets and repurposed therapies for use in SLE. Both Mariana Kaplan (National Institute of Arthritis and Musculoskeletal and Skin Diseases) and Laurence Morel (University of Florida) demonstrated how targeting immune metabolism, specifically mitochondrial respiration, could improve disease burden in patients with SLE. Morel presented data for both humans and mice with SLE, showing that metformin, widely used to treat type 2 diabetes, reduced prednisone use and decreased the number of flares in $\mathrm{SLE}^{15}$. Metformin's debated mechanisms of action not only affect the AMPK-mTOR-STAT3 pathway, but also, potentially, the electron transport chain, by inhibiting complex I. As a result, metformin reduces oxidative phosphorylation in $\mathrm{CD}^{+} \mathrm{T}$ cells isolated from patients with lupus and decreases IFN- $\gamma$ production. This finding was recapitulated in Sle1Sle2Sle 3 mice, but effects varied in other lupus mouse models, which is potentially indicative of strain-specific mechanisms involving mitochondrial respiration ${ }^{16}$.

Another repurposed drug, presented by Kaplan, demonstrated the benefit of targeting mitochondrial dysfunction to reduce aberrant apoptosis that occurs in SLE. Used in clinical trials for muscular dystrophies and neuropathies, idebenone acts as an antioxidant that bypasses complex I activity and enhances ATP synthesis. Without suppressing the immune system, idebenone improved renal disease in both the MRL/lpr and NZM2328 strains, decreased mitochondrial ROS production and neutrophil extracellular trap formation, and attenuated vasculopathy ${ }^{17}$. Similar findings were observed with the use of Mito-Q, another mitochondrial antioxidant, in MRL/lpr mice. These findings, along with Morel's, demonstrate the potential of targeting immunometabolism in SLE.

The prospect of using anti-hypertensive angiotensinogen-converting enzyme (ACE) inhibitors to treat neuropsychiatric lupus manifestations was presented by Betty Diamond (Feinstein Institute). Building upon increased brain parenchymal ACE expression in lupus mice, Diamond demonstrated that administration of ACE inhibitors led to decreased microglial activation, prevented dendritic loss by increasing expression of the inhibitory receptor LAIR-1 and improved cognition in lupus mice ${ }^{18}$. Furthermore, in conjunction with human evidence indicating that ACE polymorphisms are associated with SLE, these findings demonstrate that modulating microglia by ACE inhibition is a beneficial repurposed therapy for patients with neuropsychiatric lupus.

Chaim Putterman (Azrieli Faculty of Medicine) discussed the potential of targeting CD6-ALCAM (activated leukocyte cell adhesion molecule) interactions, which are involved in the costimulation and activation of $\mathrm{T}$ cells as well as the trafficking of effector $\mathrm{T}$ cells into tissues. 


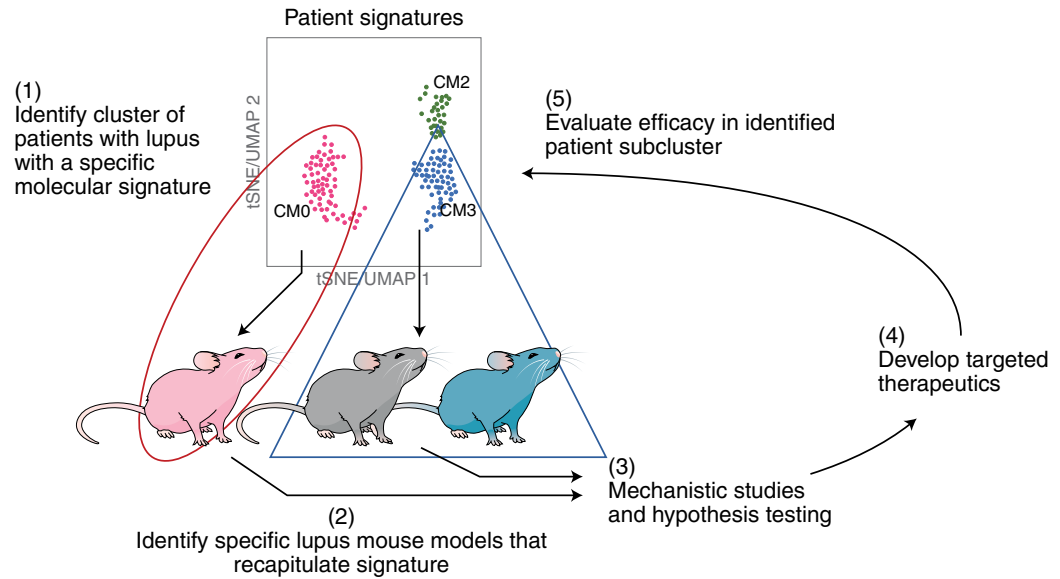

Fig. 3 | Using overlapping disease signatures in appropriate mouse models and subclusters of patients with lupus for the development of targeted therapies. With advanced technology, it is possible to select specific mouse models that recapitulate the molecular features found in subgroups of patients with lupus. The insights and therapies resulting from studying these high-fidelity models should be translated back to their matching patient subgroups, increasing the likelihood of improving patient care.

Itolizumab is a monoclonal antibody targeting CD6 that blocks CD6-ALCAM interactions without depleting T cells. In patients with SLE, urinary ALCAM concentrations are elevated, particularly in those with active lupus nephritis, and are strongly correlated with SLE disease activity index scores, glomerular filtration rate and complement concentrations, and thus could serve as a potential biomarker for stratifying patients ${ }^{19}$. In MRL/lpr mice, anti-CD6 treatment improved renal disease, survival and extra-renal disease, such as skin histopathology ${ }^{20}$. Subsequently, a phase $1 \mathrm{~b}$ clinical trial with itolizumab has been initiated for SLE and lupus nephritis, in which urinary ALCAM and/or CD6 will be followed to determine whether these biomarkers can predict responsiveness to itolizumab (NCT04128579).

Recent advances have not only identified new drug targets but have also elucidated potential adverse mechanisms contributing to drug-induced lupus or autoimmunity, as has been observed for tumor necrosis factor (TNF) inhibitors. Tam Quach (Feinstein Institute) highlighted the varying mechanisms leading to autoantibody production in different mouse models lacking TNF, including extrafollicular pathways, the induction of memory cells, and aberrant germinal center formation. Quach suggested that TNF may also influence negative regulatory $\mathrm{B}$ cell functions, and thus its deletion results in a change in the threshold for the deletion of autoreactive $B$ cells, leading to disease acceleration and increased mortality in permissive models.
Across human and mouse SLE data, there are a number of emerging drug targets, new or repurposed, that show promise and should be further evaluated. Furthermore, additional mouse model studies may reveal select stages in lupus in which specific therapies would have the greatest beneficial effect.

\section{New technologies}

In the past 10 years, innovative techniques such as the CRISPR-Cas9 system and single-cell RNA sequencing have broadly improved the ability to test hypotheses in disease models, shed new light on established concepts in SLE, and highlight the similarities and differences between lupus mouse models and patients with SLE. Eric Meffre (Yale University) investigated the mechanisms contributing to defective central B cell tolerance by using humanized mice grafted with hematopoietic stem cells $^{21}$. In particular, the role of the tyrosine phosphatase PTPN22, encoded by a risk allele in SLE, was evaluated for its potential contribution to the loss of tolerance. Interestingly, blockade of PTPN22 in mice humanized with SLE hematopoietic stem cells corrected the loss of central tolerance even when the risk allele was absent. Meffre suggested that increased receptor editing may account for the improvement.

In discerning the mechanisms contributing to skin manifestations in lupus, Mitra Maz (University of Michigan) demonstrated differential type I IFNdependent immune responses in the skin of lupus mice following UVB exposure, leading to the subsequent recruitment of monocyte and macrophage populations. This work complements previous reports that even lupus keratinocytes from non-lesional skin display a type I IFN signature ${ }^{22}$, thus highlighting the complex interplay between IFNs and both immune and stromal cells in the pathogenesis of discoid lupus.

Another emerging avenue for research includes the evaluation of altered homeostatic pathways, such as circadian rhythms, in lupus. Anne Davidson (Feinstein Institute) presented on how the kidney-specific circadian rhythm of renal homeostatic functions, such as metabolism and blood pressure regulation, are disrupted in nephritic $\mathrm{NZB} \times \mathrm{W} / \mathrm{F} 1$ lupus mice. Once remission is induced, partial corrections in glycolysis, vascular remodeling and regeneration and reversal of the abnormal blood pressure dipping pattern were observed in these mice, causing their reversion to a younger, healthier phenotype ${ }^{23}$. Davidson suggested that renal circadian rhythms, including urinary electrolyte excretion and blood pressure, measured through ambulatory blood pressure monitoring, could be tested as disease biomarkers and may also help dictate when specific therapeutics should be administered to correspond with a target organ's internal processes.

Deepak Rao (Brigham and Women's Hospital) highlighted work from the Accelerating Medicines Partnership network characterizing the adaptive immune cells in the kidneys of patients with lupus nephritis and discussed the expansion of $\mathrm{CD} 4^{+} \mathrm{T}$ cells among peripheral blood mononuclear cells from patients with SLE. PD- $1^{\text {hi } C X C R 5 ~}$ peripheral helper $\mathrm{T}\left(\mathrm{T}_{\mathrm{PH}}\right)$ cells were identified and shown to mediate $\mathrm{B}$ cell help in a manner dependent on the transcription factor $\mathrm{MAF}^{24}$. With a similar transcriptomic signature as that in $\mathrm{T}_{\mathrm{FH}}$ cells, emerging evidence has identified the presence of $\mathrm{T}_{\mathrm{PH}}$ cells in the pristane-induced lupus model. A benefit of mouse models highlighted by Rao is the ability to use the CRISPR-Cas9 and Cre/loxP systems to elucidate the various contributions similar and/or unique $\mathrm{T}_{\mathrm{FH}}$ and $\mathrm{T}_{\mathrm{PH}}$ cell markers make to the cell's function.

Further functional characterization of $\mathrm{T}_{\mathrm{FH}}$ cells in SLE indicated the enhanced role of STAT4 in the secretion of IL-21 and IFN- $\gamma$. Jason Weinstein (Rutgers University) demonstrated that mouse lupus $\mathrm{T}_{\mathrm{FH}}$ cells develop a hyper-responsiveness to STAT4 activation over time that maintains cytokine production, despite no observed increase in the expression of either IFN receptors or STAT4 itself $^{25}$. Similar results were observed in a cluster of circulating $\mathrm{T}_{\mathrm{FH}}$-like cells in patients with lupus, along with the association of increased STAT4 activation with disease severity. 
Erica Moore (Albert Einstein College of Medicine) highlighted similarities between the mouse and human $\mathrm{T}$ cell receptor (TCR) repertoires in SLE. Using CDR3 sequencing, enhanced sample clonality demonstrated the directed immune response present in patients with SLE as compared to healthy controls. Additional analysis of the TCR repertoires revealed a skewed use of $\mathrm{V}$ genes in both humans and mice and the potential to use select TCR sequences as biomarkers for diagnostic or prognostic purposes in patients with SLE ${ }^{26}$. Furthermore, select TCR sequences alongside T cell phenotyping could be used to monitor specific organ manifestations (Fig. 2), as highlighted by an identified consensus sequence in brain-infiltrating T cells in MRL/lpr mice with neuropsychiatric manifestations ${ }^{27}$.

Paul Hoover (Brigham and Women's Hospital and Broad Institute) compared human SLE signatures and lupus mouse models using single-cell RNA sequencing. The characterization of myeloid cells in the kidneys of mice with lupus revealed that the main populations were residential macrophages, classical and non-classical monocytes and circulating dendritic cells in both Sle1Yaa and NZB/W models ${ }^{28}$. Based on differentially expressed gene profiles, a number of the myeloid signatures present in humans, excluding the resident macrophage cluster, were observed in the two mouse lupus models, and some were associated with nephritis. Furthermore, Hoover showed preliminary data suggesting that the monocyte subsets occupied similar kidney compartments in both mouse and human kidney tissue. Differences between the lupus mouse strains were suggested to mirror patient-specific phenotypes as well as different aspects of disease.

Improved methodologies can more readily elucidate mechanisms in greater detail and with nuance and can identify mouse models that may best match subsets of patients with SLE. While no singular mouse model recapitulates lupus disease in humans, these recent studies highlight overlapping mechanisms between patients with SLE and mouse models and the possibility of selectively using mouse models to appropriately query facets of SLE pathogenesis and develop new treatments (Fig. 3).

\section{Concluding remarks}

The meeting gathered early career and established scientists and clinicians to debate and discuss the usefulness of lupus mouse models. Through human and mouse data comparisons as well as lengthy discussions, the data presented showed that lupus mouse models still perform an irreplaceable function in modelling systemic autoimmunity and elucidating facets of disease pathogenesis. More specifically, experimental manipulations in these models have accelerated various avenues of research, including identifying internal triggers, via alterations in the microbiome; target-organ-specific mechanisms; and emerging drug targets, such as immunometabolism. Recognizing the heterogeneity of lupus manifestations and likely disease mechanisms, the consensus was to continue using lupus mouse models but to do so in a purposeful and directed manner, testing specific questions relevant to the particular model. Furthermore, technological advances will enhance our ability to first use findings in particular subsets of patients with SLE, which will guide the selection of mouse models and experiments in developing targeted therapies for the clinic.

\section{Erica Moore (D) 1ه, Joshua A. Reynolds (D), Anne Davidson², Stefania Gallucci3 ${ }^{3}$, Laurence Morel (D) 4 , Deepak A. Rao(iD), Howard A. Young (D) 6 and \\ Chaim Putterman ${ }^{1,7,8}$ \\ ${ }^{1}$ Albert Einstein College of Medicine, Bronx, NY, USA. ${ }^{2}$ Feinstein Institutes for Medical Research, Manhasset, NY, USA. ${ }^{3}$ Lewis Katz School of Medicine, Temple University, Philadelphia, PA, USA. ${ }^{4}$ University of Florida College of Medicine, Miami, FL, USA. ${ }^{5}$ Brigham and Women's Hospital and Harvard Medical School, Boston, MA, USA. ${ }^{6}$ National Cancer Institute, Frederick, MD, USA. ${ }^{7}$ Azrieli Faculty of Medicine of Bar-Ilan University, Safed, Israel. ${ }^{8}$ Galilee Research Institute, \\ Nahariya, Israel. \\ $凶_{e-m a i l: \text { erica.moore@einsteinmed.org }}$}

Published online: 10 May 2021

https://doi.org/10.1038/s41590-021-00914-4

References

1. Moore, E. \& Putterman, C. J. Autoimmun https://doi.org/10.1016/j.jaut.2020.102490 (2020).

2. Richard, M. L. \& Gilkeson, G. Lupus Sci. Med. https://doi.org/10.1136/lupus-2016-000199 (2018).

3. Li, W., Titov, A. A., Morel, L. Curr. Opin. Rheumatol. https://doi.org/10.1097/BOR.0000000000000412 (2017).

4. Di Niro, R. et al. Immunity https://doi.org/10.1016/j. immuni.2015.06.013 (2015)

5. Elsner, R. A. \& Shlomchik, M. J. Cell Rep. https://doi. org/10.1016/j.celrep.2019.10.069 (2019).

6. Miller, A. L. et al. PLoS Pathog. https://doi.org/10.1371/journal. ppat.1008591 (2020)
7. Gallo, P. M. et al. Immunity https://doi.org/10.1016/j. immuni.2015.06.002 (2015)

8. Pachucki, R. J. et al. Arthritis Rheumatol. https://doi.org/10.1002/ art.41400 (2020).

9. Azzouz, D. et al. Ann. Rheum. Dis. https://doi.org/10.1136/ annrheumdis-2018-214856 (2019).

10. Manfredo Vieira, S. et al. Science https://doi.org/10.1126/science. aar7201 (2018).

11. Zhang, H., Liao, X., Sparks, J. B. \& Luo, X. M. Appl. Environ. Microbiol. https://doi.org/10.1128/AEM.02676-14 (2014).

12. Mu, Q. et al. Microbiome https://doi.org/10.1186/s40168-0170300-8 (2017).

13. Mu, Q. et al. Sci. Rep. https://doi.org/10.1038/s41598-017-14223-0 (2017).

14. Zegarra-Ruiz, D. F. et al. Cell Host Microbe https://doi.org/ 10.1016/j.chom.2018.11.009 (2019).

15. Sun, F. et al. Lancet Rheumatol. https://doi.org/10.1016/S26659913(20)30004-7 (2020).

16. Titov, A. A., Baker, H. V., Brusko, T. M., Sobel, E. S. \& Morel, L. J. Immunol. https://doi.org/10.4049/jimmunol.1801651 (2019).

17. Blanco, L. P. et al. Arthritis Rheumatol. https://doi.org/10.1002/ art.41128 (2020).

18. Nestor, J. et al. J. Exp. Med. https://doi.org/10.1084/jem.20180776 (2018).

19. Stanley, S. et al. Nat. Commun. https://doi.org/10.1038/s41467 020-15986-3 (2020)

20. Chalmers, S. et al. Arthritis Rheumatol. 71 (Suppl. 10), abstr. 65 (2019).

21. Schickel, J.-N. et al. Sci. Immunol. https://doi.org/10.1126 sciimmunol.aaf7153 (2016).

22. Der, E. et al. Nat. Immunol. https://doi.org/10.1038/s41590-0190386-1 (2019).

23. Misha, R. et al. Arthritis Rheumatol. 71 (Suppl. 10), abstr. 1783 (2019).

24. Bocharnikov, A. V. et al. JCI Insight https://doi.org/10.1172/jci. insight.130062 (2019).

25. Dong, X. et al. Arthritis Rheumatol. https://doi.org/10.1002/ art.41532 (2021).

26. Liu, X. et al. Ann. Rheum. Dis. https://doi.org/10.1136/ annrheumdis-2019-215442 (2019).

27. Moore, E. et al. Front. Immunol. https://doi.org/10.3389/fimmu. 2020.01476 (2020).

28. Hoover, P. et al. Arthritis Rheumatol. 72 (Suppl. 10), abstr. 979 (2020).

Acknowledgements

We thank all of the speakers for participating in the meeting's robust discussion on the usefulness of mouse models in lupus research. Additionally, we thank Jose Aragon, Luis Mendez, and the rest of the IT team at the National Cancer Institute (NCI) for their support both prior to and on the day of the meeting. This work was supported by the Intramural Research Program of the Center for Cancer Research, NCI. The views expressed in this article are those of the authors and do not necessarily reflect the official policy or position of the Department of Health and Human Services, nor does mention of trade names, commercial products, or organizations imply endorsement by the United States Government. Funding support included T32-GM007288 from the National Institute of General Medical Sciences (E.M. and J.A.R.); R21AR076557 (A.D.) and K08AR072791 (D.A.R.) from the National Institute of Arthritis and Musculoskeletal and Skin Diseases (A.D); W81XWH-17-1-0657 from the Department of Defense, Lupus Research Alliance (A.D., S.G., and D.A.R.); R01AI128901 from the National Institute of Allergy \& Infectious Diseases (L.M.); and a Burroughs Wellcome Fund Career Award in Medical Sciences (D.A.R.).

Competing interests

C.P. is a consultant to Equillium and is one of the investigators on the current anti-CD6 antibody treatment trial for lupus. The other authors declare no competing interests. 